EPJ Web of Conferences 103, 01009 (2015)

DOI: $10.1051 /$ epjconf/ 201510301009

(C) Owned by the authors, published by EDP Sciences, 2015

\title{
Dynamical Lamb Effect: Prediction and Possibility of Experimental Detection
}

\author{
Yu.E. Lozovik ${ }^{1,2 a}$, D.S. Shapiro ${ }^{2,3}$, A.A. Zhukov ${ }^{2,4}$, and W.V. Pogosov ${ }^{2,5}$ \\ ${ }^{1}$ Institute of Spectroscopy, Russian Academy of Sciences, 142190 Moscow region, Troitsk, Russia \\ ${ }^{2}$ Center for Fundamental and Applied Research, N.L. Dukhov All-Russia Research Institute of Automatics, 127055 \\ Moscow, Russia \\ ${ }^{3}$ V.A.Kotel'nikov Institute of Radio Engineering and Electronics, Russian Academy of Sciences, 125009 Moscow, \\ Russia \\ ${ }^{4}$ National Research Nuclear University (MEPhl), 115409 Moscow, Russia \\ ${ }^{5}$ Institute for Theoretical and Applied Electrodynamics, Russian Academy of Sciences, 125412 Moscow, Russia
}

A natural atom placed into a cavity with time-dependent parameters can be parametrically excited due to the interaction with the quantized photon mode. One of the channels of such a process is the dynamical Lamb effect, induced by a nonadiabatic modulation of atomic level Lamb shift. However, in experiments with natural atoms it is quite difficult to isolate this effect from other mechanisms of atom excitation. We point out that a transmission line cavity coupled with a superconducting qubit (artificial macroscopic atom) provides a unique platform for the observation of the dynamical Lamb effect. A key idea is to exploit a dynamically tunable qubit-resonator coupling, which was implemented quite recently. By varying nonadiabatically the coupling, it is possible to parametrically excite a qubit through a nonadiabatic modulation of the Lamb shift, even if the cavity was initially empty. A dynamics of such a coupled system is studied within the Rabi model with timedependent coupling constant and beyond the rotating wave approximation. An efficient method to increase the effect through the periodic and nonadiabatic switching of a qubit-resonator coupling energy is proposed.

Superconducting circuits with Josephson junctions can be used for quantum computing as qubits. They can be integrated with microwave waveguides, while the photon field in these waveguides is quantized. A well known Rabi model is applicable for the description of qubit-photon quantum system.

Moreover, superconducting circuits integrated with microwave resonators provide a unique platform for an observation of cavity quantum electrodynamical (QED) nonstationary phenomena which can hardly be studied in more traditional experiments. One of the examples of such nonstationary QED phenomena is the dynamical Casimir effect. It is remarkable that it was a superconducting system, which led to the first observation of the dynamical Casimir effect $[1,2]$.

A presence of an additional atom in a cavity with time-dependent parameters leads to other nonstationary QED phenomena. For instance, an atom can be parametrically excited, even if the cavity was initially empty. In the case of a nonadiabatical modulation of cavity parameters, there are

\footnotetext{
${ }^{\text {a } C o r r e s p o n d i n g ~ a u t h o r: ~ l o z o v i k @ i s a n . t r o i t s k . r u ~}$
} 
two channels of such a process [3]. The most obvious mechanism is due to the absorbtion of Casimir photons. Another channel is due to the nonadiabatic modulation of atomic Lamb shift: virtual states of atom-photon coupled system are transformed into real states. This phenomenon can be called a dynamical Lamb effect. In practice, it is quite difficult to isolate the mechanism of atom excitation due to the dynamical Lamb effect from other channels.

The main goal of this work is to suggest a superconducting qubit-resonator system as a suitable platform for the observation of the dynamical Lamb effect instead of a natural atom-cavity system. An astonishing property of superconducting qubits is a high tunability of their parameters in situ during an experiment. In particular, it becomes realistic to vary dynamically not only the resonator frequency, but also a coupling between the artificial atom and photon field with the frequency of a resonator or even faster [4]. Thus, we consider a qubit initially uncoupled from the resonator, while at certain moment this coupling is nonadiabatically switched on. This operation is accompanied by a nonadiabatical modulation of the Lamb shift and thus should lead to the dynamical Lamb effect.

In order to describe theoretically the dynamical Lamb effect in the system with the tunable qubitphoton coupling, we use the Rabi model, in which we have to go beyond the rotating wave approximation (RWA) in order to take into account counter-rotating processes. These are the photon creation with the simultaneous qubit excitation and the opposite process. We take into account these processes by constructing a perturbation theory around the stationary RWA solution. In addition, we solve a time-dependent Schrödinger equation numerically. The total Hamiltonian takes into account photons at the single mode $\omega$ and the qubit with the bare excitation frequency $\epsilon$ with the coupling $V$ between them

$$
H=\omega a^{\dagger} a+\frac{1}{2} \mathrm{~T}\left(1+\sigma_{3}\right)+\mathrm{V},
$$

where $a^{\dagger}$ and $a$ a are secondary quantized boson operators of the photon field, while Pauli matrices $\sigma_{3}=2 \sigma_{+} \sigma_{-}-1, \sigma_{+}, \sigma_{-}$act in the space of qubit states. The operator $V$ reads as

$$
\mathrm{V}=g\left(a+a^{\dagger}\right)\left(\sigma_{-}+\sigma_{+}\right)
$$

It can be split as

$$
\mathrm{V}=V_{1}+V_{2}
$$

where

$$
\mathrm{V}_{1}=g\left(a \sigma_{+}+a^{\dagger} \sigma_{-}\right)
$$

is the rotating wave contribution, conserving the excitation number, and

$$
\mathrm{V}_{2}=g\left(a^{\dagger} \sigma_{+}+a \sigma_{-}\right)
$$

is the counter-rotating term, which breaks this symmetry, but conserves a parity.

We assume that in the initial moment, the qubit and the resonator were not coupled, so that the initial state of the whole system was $|0 \downarrow\rangle$. We then study the evolution of the wave function in a full resonance regime, $\epsilon=\omega$, after the sudden switch of the coupling $g$, which then stays constant in time. In the first-order approximation, the qubit excitation probability is given by

$$
w_{\uparrow}(\mathrm{t})=\frac{g^{2}}{8 \omega^{2}}(3+\cos 2 \sqrt{2} g t-4 \cos 2 \omega t \cos \sqrt{2} g t) .
$$

This probability corresponds to the dynamical Lamb effect and includes both slow and fast oscillations. In the superconducting systems with weak coupling between photon field and qubit, it is as small as $g^{2} / \omega^{2}$.

We also point out that the excitation probability can be dramatically increased at the regime of a parametric resonance, when the driving is induced by high frequency periodic switching of the qubitresonator coupling. In particular, we suggest applying $2 \omega$-periodical switching on and off, namely, $g(\mathrm{t})=\mathrm{g} \theta(\cos 2 \omega t)$. The corresponding excitation probability is 


$$
w_{\uparrow}(t) \cong \frac{2}{\pi^{2}}(1-\cos 2 \sqrt{2} g t)
$$

This result describes an approximate Rabi-like oscillatory behavior of the qubit excitation probability via the dynamical Lamb effect in the regime of a parametric resonance. Oscillations of the same type appear also for various photon characteristics of the system. Regardless of the relation between amplitude of the switching $g$ and $\omega$, the maximum probability reaches the finite value $4 / \pi^{2}$ on a characteristic time scale $\sim 1 / g$, which depends on the amplitude of the inverse coupling strength only. In contrast to the probability in the regime of a single switching (6), the time dependence of $w_{\uparrow}(t)$ in parametric resonance regime (7) does not contain high-frequency oscillations at $\omega$. Moreover, the maximum probability is not small anymore. This last feature is of great importance for the possibility of an experimental investigation of the dynamical Lamb effect. We also wish to stress that the precise value of the maximum excitation probability, as given by Eq. (6), is rather relative. Nevertheless, Eq. (7) yields correct qualitative description for $w_{\uparrow}(t)$, as shown by a comparison with fully numerical results.

Slow Rabi-like oscillations of various characteristics of a photon field can be used in experiments as a signature of the dynamical Lamb effect. Indeed, if the resonator was initially empty and qubit was uncoupled from it, no such oscillations can exist. If a coupling energy is modulated, qubit can be excited leading to photon creation via counter-rotating processes, which can be seen in experiments. In contrast to the dynamical Casimir effect, photons occupy not only even states.

\section{References}

1. C. M. Wilson, G. Johansson, A. Pourkabirian, J. R. Johansson, T. Duty, F. Nori, and P. Delsing, Nature 479, 376 (2011)

2. P. Lähteenmäki, G. S. Paraoanu, J. Hassel, and P. J. Hakonen, Proc. Natl. Acad. Sci. U.S.A. 110, 4234 (2013)

3. N. B. Narozhny, A. M. Fedotov, and Yu. E. Lozovik, Phys. Rev. A 64, 053807 (2001)

4. Yu Chen, C. Neill, P. Roushan, N. Leung, M. Fang, R. Barends, J. Kelly, B. Campbell, Z. Chen, B. Chiaro, A. Dunsworth, E. Jeffrey, A. Megrant, J. Y. Mutus, P. J. J. O'Malley, C. M. Quintana, D. Sank, A. Vainsencher, J. Wenner, T. C. White, M. R. Geller, A. N. Cleland, and J. M. Martinis, Phys. Rev. Lett. 113, 220502 (2014) 